\title{
K-means-net: Clusterização e escolha de gateways em redes de sensores sem fio
}

\section{K-means-net: Clustering and choosing gateways on wireless sensor networks}

Larissa Maria Santiago Correia ${ }^{1}$, Alexandre Celestino Leite Almeida², Marcos Tomio Kakitani ${ }^{3}$

\section{RESUMO}

O posicionamento de nós gateways em uma rede de sensores sem fio com múltiplos saltos tem impacto direto na qualidade dos serviços da rede. Uma boa escolha dos gateways resulta em um menor número de saltos entre um nó e seu gateway (GW) correspondente na rede, causando menos perdas, menor tempo de comunicação e melhorando a qualidade de serviço. O tamanho da rede pode tornar o problema de encontrar soluções ótimas para a implantação dos gateways bastante complexo. Este trabalho propõe um algoritmo baseado no método K-means em conjunto com teoremas de substituição com a finalidade de dividir a rede em $K$ sub-redes e na escolha do GW de cada uma delas. Os teoremas de substituição são usados para encontrar nós não redundantes, possíveis gateways, em cada cluster dividido pelo K-means. A adaptação da utilização simultânea destes dois métodos fornece boas soluções para a implantação dos GWs na rede, bem como já fornece o conjunto de nós de cada GW.

Palavras-chave: T-step. K-means. RSSF. Rede de sensores sem fio. Teoria de grafos.

\section{ABSTRACT}

The positioning of gateways nodes in a multi-hop wireless sensor network has a direct impact on the quality of network services. A good choice of gateways results in fewer hops between a node and its corresponding gateway (GW) in the network, causing fewer losses, shorter communication time, and improved quality of service. The size of the network can make the problem of finding optimal solutions for the deployment of gateways quite complex. This work proposes an algorithm based on the K-means method in conjunction with substitution theorems, with the purpose of dividing the network into K subnets and choosing the GW of each of them. Substitution theorems are used to find non-redundant nodes, possible gateways, in each cluster divided by Kmeans. The adaptation of the simultaneous use of these two methods provides good solutions for the deployment of the GWs in the network, as well as already provides the set of nodes of each GW.

Keywords: T-step. K-means. WSN. Wireless sensor networks. Graph theory.
${ }^{1}$ Graduanda do curso de

Engenharia de

Telecomunicações da UFSJ -

Campus Alto Paraopeba, Ouro

Branco-MG.

E-mail:

larissa.mscorreia@gmail.com

${ }^{2}$ Docente do departamento de Física e Matemática da UFSJ Campus Alto Paraopeba, Ouro Branco-MG.

${ }^{3}$ Docente do departamento das Engenharias de

Telecomunicações e Mecatrônica da UFSJ - Campus Alto

Paraopeba, Ouro Branco-MG. 


\section{INTRODUÇAOO}

As Redes de Sensores Sem Fio (RSSF) são um tipo de rede móvel com a finalidade de monitorar eventos e transmitir as informações coletadas através de comunicação sem fio. O monitoramento é feito por meio de pequenos componentes, chamados nós sensores. Estes nós podem se comunicar entre si ou diretamente com um Gateway (GW), o qual é responsável pela coleta de dados e envio para algum centro de dados externo à rede. Um GW pode ser externo à rede ou um nó da mesma. Neste trabalho, estamos interessados no problema de escolher como GW um nó pertencente à rede. Tipicamente, os nós sensores são compostos por elementos que possuem limitações quanto às suas fontes de energia, podendo apresentar restrições ou até mesmo inviabilidade em relação à recarga de suas baterias ou substituição dos nós. Desta forma, minimizar o número de saltos entre os nós e os GWs é muito importante em uma RSSF.

Uma rede de sensores sem fio (RSSF) de grande volume representa um problema para o melhor posicionamento dos sensores que vão transmitir ou recolher informações desta rede. Tendo em vista o diminuto tamanho dos nós, a duração da bateria dos mesmos depende do volume de dados que passa por ele.

Um dos principais problemas para a implantação de gateways (GWs) em uma RSSF é escolher um nó que diminua o maior número de saltos das informações para transmissão de dados. Desta maneira, o fluxo de dados em um mesmo nó é diminuído e a bateria do mesmo dura por mais tempo, possibilitando maior tempo de coleta de dados.

Dividir uma rede grandiosa em sub-redes menores, cada uma com um GW responsável pela coleta ou transmissão de dados de todos os nós associados àquela subrede é um modo estudado para a melhor implantação destes GWs. Tais sub-redes são chamadas de clusters.

Empregar métodos inteligentes, tais como otimização por enxame de partículas (PSO), computação evolucionária (EC), otimização de colônias de formigas (AIA), K-means, algoritmos genéticos (GA) com diferentes abordagens e outros exemplificados em (REBAI et al., 2015), (MÁGAN-CARRIÓN et al., 2016), (LIN, 2013), (LIN et al., 2016), (YOON e KIM, 2013) e (ABDELKHALEK et al., 2015) são estratégias bastante utilizadas para a otimização de redes sem fio.

O presente trabalho propõe para a resolução do problema de implementação dos gateways um método inteligente, o K-means adaptado, em conjunto com alguns teoremas, apresentados em (HUANG et al., 2017). O método é aplicado para o clustering da rede. 0 
nó centro do cluster é definido como aquele que resolve o problema de otimização da Equação 1 dentro do cluster e é obtido baseado nos teoremas, chamados de teorema da substituição e teorema do $t$-step substituição.

Estes teoremas permitem eliminar os nós redundantes dos clusters, ou seja, eliminam nós que possuem escolhas melhores de GW na rede, diminuindo o espaço de busca pelo GW dentro de cada cluster e aumentando a probabilidade de chegar a soluções ótimas.

Neste trabalho consideraremos que as distâncias em saltos entre quaisquer dois nós são sempre distâncias mínimas. Para otimizar a implementação de gateways nosso objetivo é minimizar os saltos da rede. Deste modo, nosso problema pode ser formulado como:

$$
\begin{aligned}
& \min \max _{1 \leq j \leq k}\left\{\max _{n_{i} \in C_{j}} d\left(u_{j}, n_{i}\right)\right\}, \\
& \text { sujeito a: }\left\{u_{1}, u_{2}, \ldots, u_{k}\right\} \subset N
\end{aligned}
$$

sendo $n_{i}$ um nó da rede, $u_{j}$ um GW, $N$ o conjunto de nós da rede, $C_{j}$ um dos clusters existentes e $d\left(u_{j}, n_{i}\right)$ a distância em saltos entre $u_{j}$ e $n_{i}$.

\section{ALGORITMOS RELACIONADOS}

Para a realização deste trabalho foi considerado um algoritmo de otimização em conjunto com alguns teoremas propostos em (HUANG et al., 2017).

\subsection{K-means}

O K-means é um bem conhecido método de agrupamento que usa técnicas estatísticas sendo um algoritmo de clusterização. Tal algoritmo encontra $K$ centros em um conjunto de nós que minimizam a distância entre cada nó e o centro mais próximo. (KANUNGO et al., 2002).

O K-means tradicional calcula centroides imaginários para cada cluster, que são a média das posições dos nós que pertencem ao mesmo. O clustering (agrupamento) é então reestabelecido e novos centroides são criados. Uma resposta satisfatória é obtida quando a posição dos centroides para de variar bruscamente.

A utilização do K-means em uma RSSF onde o GW deve ser um dos nós já existentes não é direta, uma vez que o K-means, em nosso problema, não terá liberdade de escolha do centro em qualquer posição do espaço. Além disso, o K-means usa a distância 
Euclidiana dos nós em seus cálculos e, em nosso problema, estamos interessados no número de saltos entre cada nó e seu GW.

Aplicado ao nosso problema, desenvolvemos um algoritmo baseado no $\mathrm{K}$-means, que divide a RSSF em $K$ clusters, de modo que nós com menos saltos até seu respectivo GW tendem a se conservar em um mesmo cluster. Os centroides deixam de ser médias das posições dos nós do cluster e se tornam nós existentes na RSSF. Os centroides finais serão os melhores nós para serem considerados gateways.

\subsection{Teorema da substituição - T-step}

Em seu trabalho recente, (HUANG et al., 2017) propuseram os teoremas da substituição. Através destes teoremas, a complexidade para encontrar boas soluções para uma RSSF composta por uma grande quantidade de nós diminui notavelmente. O espaço de busca é reduzido significativamente com a aplicação dos teoremas, que resultam na melhora do desempenho dos algoritmos que fazem o uso de tais artifícios.

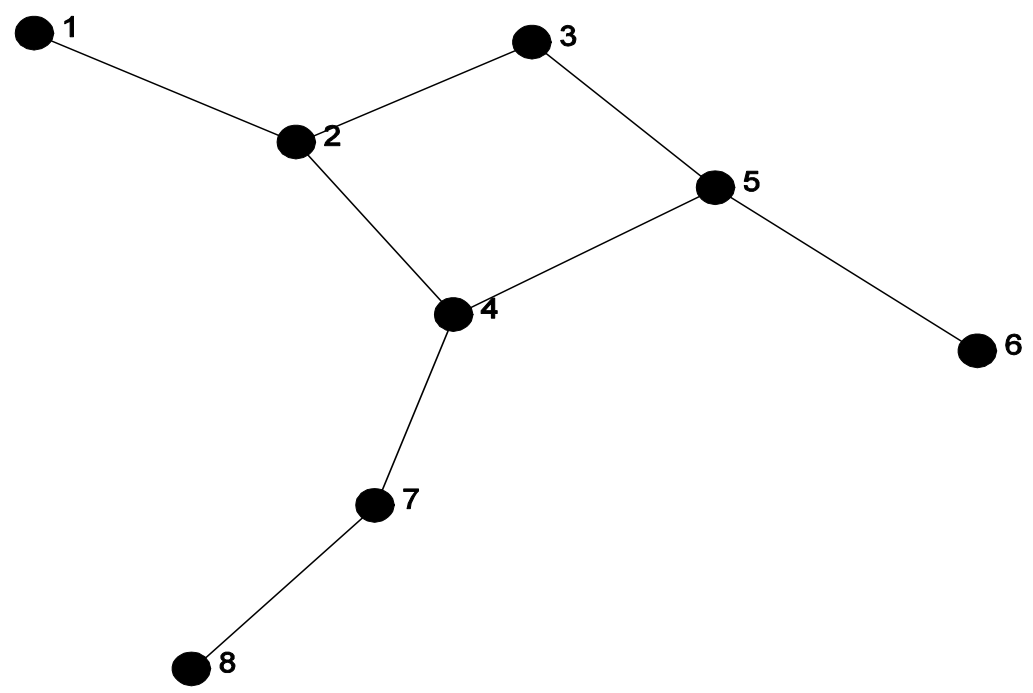

Figura 1. Exemplo de GD para uma rede sem fio com 8 nós.

Modelamos uma rede de sensores sem fio através do Grafo de Demonstração (GD), ilustrado na Figura 1. Definimos $G D=\{N, A\}$, de maneira que $N$ é o conjunto de vértices, que representam os nós presentes no grafo e que são possíveis GWs e $A$ é o conjunto de arestas do grafo que representam a distância de um salto entre os dois nós conectados. Definimos $d\left(n_{i}, n_{j}\right)$ como a distância em saltos entre dois vértices. Esta distância é o número mínimo de arestas que separam dois nós.

Para a explicação dos teoremas, são considerados alguns conceitos, explanados a seguir. 
Nó folha: são considerados nós folhas aqueles sensores que possuem conexão com apenas um nó de toda rede. Claramente, um nó folha não é um bom candidato a gateway e, portanto, pode ser excluído do espaço de busca. Considerando a Figura 1, os nós $n_{1}, n_{6}$ e $n_{8}$ possuem conexão apenas com os nós $n_{2}, n_{5}$ e $n_{7}$, respectivamente. Desta maneira, são considerados nós folha.

Nós adjacentes: Considere um nó $n_{i}$. Os nós adjacentes ao mesmo são aqueles separados por apenas uma aresta, ou seja, estão a distância de apenas um salto. Exemplificando, na Figura 1, o conjunto de nós adjacentes ao nó $n_{4}, A\left(n_{4}\right)$, é composto pelos vértices $\left\{n_{2}, n_{5}, n_{7}\right\}$, pois $d\left(n_{4}, n_{2}\right)=d\left(n_{4}, n_{5}\right)=d\left(n_{4}, n_{7}\right)=1$.

T-hop nós adjacentes: O conjunto $A_{t}\left(n_{i}\right)$ de um nó inclui todos os nós que estão a uma distância menor ou igual a $t$ saltos de $n_{i}$. A título de exemplo, considerando $t=2,0$ vértice $n_{3}$ possui $A_{2}\left(n_{3}\right)=\left\{n_{1}, n_{2}, n_{4}, n_{5}, n_{6}\right\}$ uma vez que $d\left(n_{3}, n_{2}\right)=d\left(n_{3}, n_{5}\right)=1<t$ e $d\left(n_{3}, n_{1}\right)=d\left(n_{3}, n_{4}\right)=d\left(n_{3}, n_{6}\right)=2=t$.

Teorema da substituição: Quando o conjunto de nós adjacentes para o nó $n_{i}$ é $A\left(n_{i}\right) \subset\left(n_{j} \cup A\left(n_{j}\right)\right), n_{i}$ é considerado redundante e pode ser substituído por $n_{j}$.

Teorema da t-step substituição: Quando o conjunto de nós $t$ - hop adjacentes para o nó $n_{i}$ é $A_{t}\left(n_{i}\right) \subset\left(n_{j} \cup A_{t}\left(n_{j}\right)\right)$ então $n_{i}$ é um nó redundante e pode ser substituído por $n_{j}$.

Estes teoremas serão aplicados na função t-step a qual será utilizada no pseudocódigo apresentado no Algoritmo 1. Os nós não redundantes encontrados serão os nossos candidatos a gateways.

\section{METODOLOGIA}

Neste trabalho propomos um algoritmo, denominado K-means-net, que escolhe, em uma determinada rede, os $K$ melhores nós para serem utilizados como gateways e atribui todos os nós para o GW mais próximo, formando assim, $K$ clusters.

A ordem de complexidade para se encontrar a melhor configuração de gateways (e seus clusters) é relativamente alta. Para tentar mensurar, quando a rede tem tamanho $n$ e o número de GWs é $K$, temos $C_{n}^{K}$ combinações possíveis. Isso significa que uma rede com 100 nós a ser dividida em 5 clusters possui na ordem de $10^{148}$ possíveis combinações. Desta forma, a busca por heurísticas eficientes é de suma importância. 
Nossa proposta é aplicar os teoremas apresentados em (HUANG et al., 2017) em uma modificação do algoritmo K-means para trabalhar em redes onde o GW necessita ser um dos nós. O valor de Ké determinado como a quantidade de gateways desejada. Quando $K$ $=1$, não há divisão de clusters, sendo apenas aplicado o $t$-step, de modo a achar o melhor nó para ser GW. Um paralelo entre os algoritmos K-means e K-means-net é realizado na Tabela 1.

Tabela 1. Paralelo entre os algoritmos $K$-means e $K$-means-net

\begin{tabular}{|c|c|}
\hline Passos do K-means & Passos do K-means-net \\
\hline 1 - Escolhe centros aleatórios & 1 - Escolhe nós aleatórios (nós centro) \\
\hline $\begin{array}{l}2 \text { - Divide os clusters pela distância } \\
\text { euclidiana aos centros }\end{array}$ & $\begin{array}{l}2 \text { - Divide os clusters pela distância em } \\
\text { saltos aos nós centro }\end{array}$ \\
\hline $\begin{array}{l}3 \text { - Escolhe novos centros (distância média } \\
\text { de todos os nós atrelados ao centroide) }\end{array}$ & $\begin{array}{l}3 \text { - Escolhe novos centros (usa o } t \text {-step para } \\
\text { encontrar os novos nós centro) }\end{array}$ \\
\hline $\begin{array}{c}4 \text { - Se não atingiu o critério de parada, volta } \\
\text { ao passo } 2 .\end{array}$ & $\begin{array}{c}4 \text { - Se não atingiu o critério de parada, volta } \\
\text { ao passo } 2 .\end{array}$ \\
\hline
\end{tabular}

O algoritmo proposto $\mathrm{K}$-means-net é descrito no Algoritmo 1. O mesmo considera, em sua inicialização, nós aleatórios como gateways. $O$ conjunto $N$ é então submetido a uma função definida como Cluster_Kmeans() que divide os nós em $K$ clusters, em relação aos GWs escolhidos. Esta divisão é realizada a partir da distância em saltos. Calcula-se a distância em saltos de cada nó para cada GW. O gateway associado a menor distância inclui o nó em seu cluster.

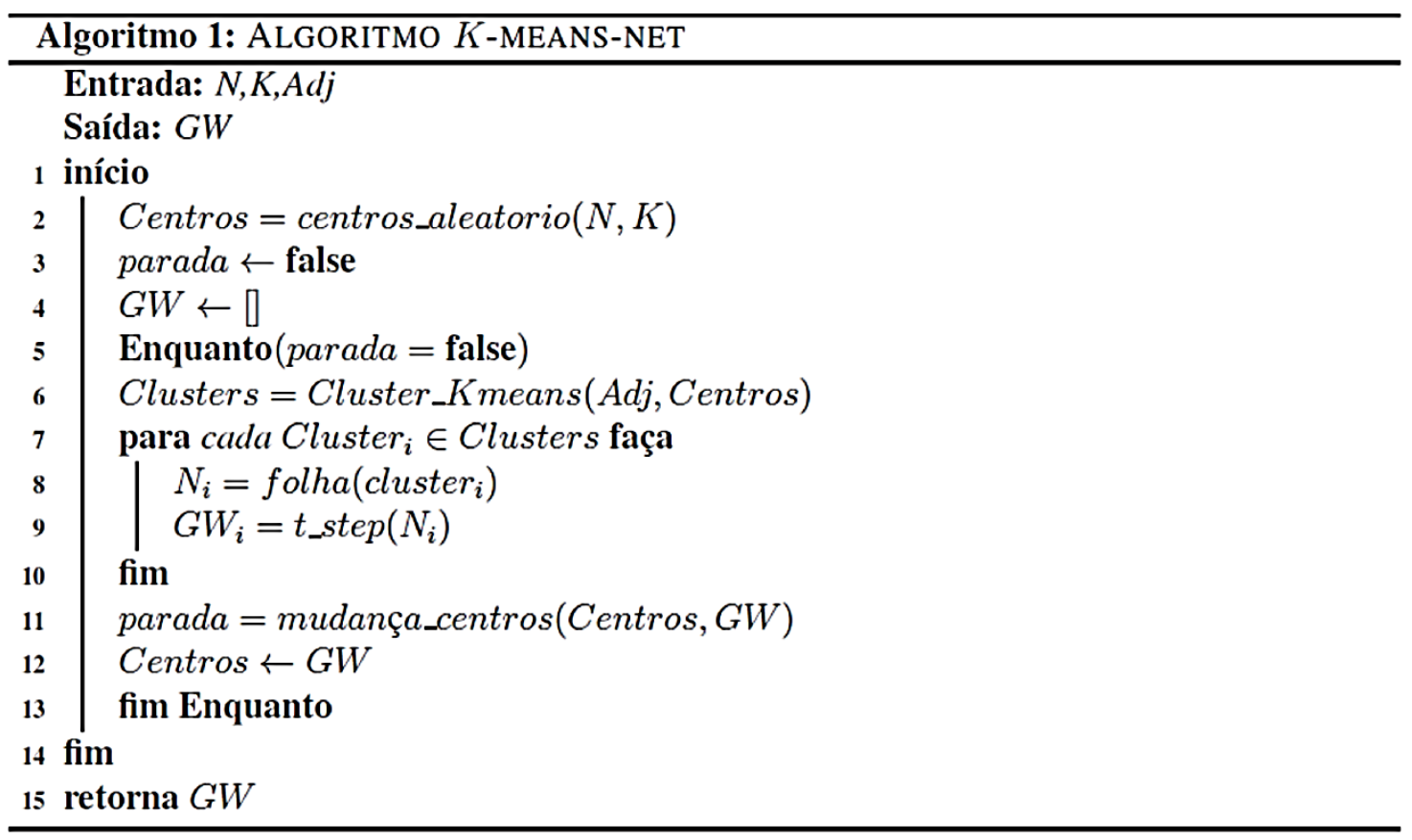

Algoritmo 1. Pseudocódigo do algoritmo proposto K-means-net 
Aplicamos então as funções folha() e $t$ _step() a cada cluster. A função folha() elimina do espaço de busca de gateways os nós folhas, uma vez que os mesmos não podem ser um nó GW. A função $t$ step() elimina do espaço de busca os nós redundantes do cluster. Os nós restantes são possíveis GWs. O nó não redundante com menor máxima distância em relação ao cluster é então escolhido como novo centroide.

A função $t \_s t e p()$ retorna os nós não redundantes da rede, ou seja, aqueles que são candidatos a GWs. A mesma recebe a matriz de adjacência $A_{t}()$ de toda a rede. Nesta matriz quadrada, cada linha e cada coluna indicam um nó. Por exemplo, se existe uma marcação na posição $(i, j)$ da matriz, isso significa que os vértices $n_{i}$ e $n_{j}$ estão conectados por alguma distância em saltos menor ou igual a $t$. As linhas são então comparadas. Se ocorre o descrito no teorema, ou seja, se $A_{t}\left(n_{i}\right)$ está contido em $n_{j} \cup A_{t}\left(n_{j}\right)$ então o nó $n_{i}$ é redundante e pode ser excluído do espaço de busca pelo GW. A função começa com $t=$ 1 e incrementa $t$ para reduzir cada vez mais o espaço de busca pelos GWs. $O$ valor de $t$ para de ser incrementado, quando $t$ se torna igual ao máximo salto da rede ou quando os candidatos a GWs se reduzem a apenas um.

Com novos centroides definidos, os clusters são recalculados e os passos são repetidos até que os centroides parem de se alterar significativamente. A função mudança_centros() avalia essa alteração.

\section{RESULTADOS}

Foram criadas redes cujas posições dos nós foram geradas aleatoriamente seguindo uma distribuição uniforme em cada coordenada e cujas arestas foram criadas apenas entre os nós que satisfazem uma distância euclidiana máxima.

A Figura 2, ilustrada a seguir, mostra o GD de uma rede aleatória com 25 nós distribuída uniformemente no plano coordenado. 


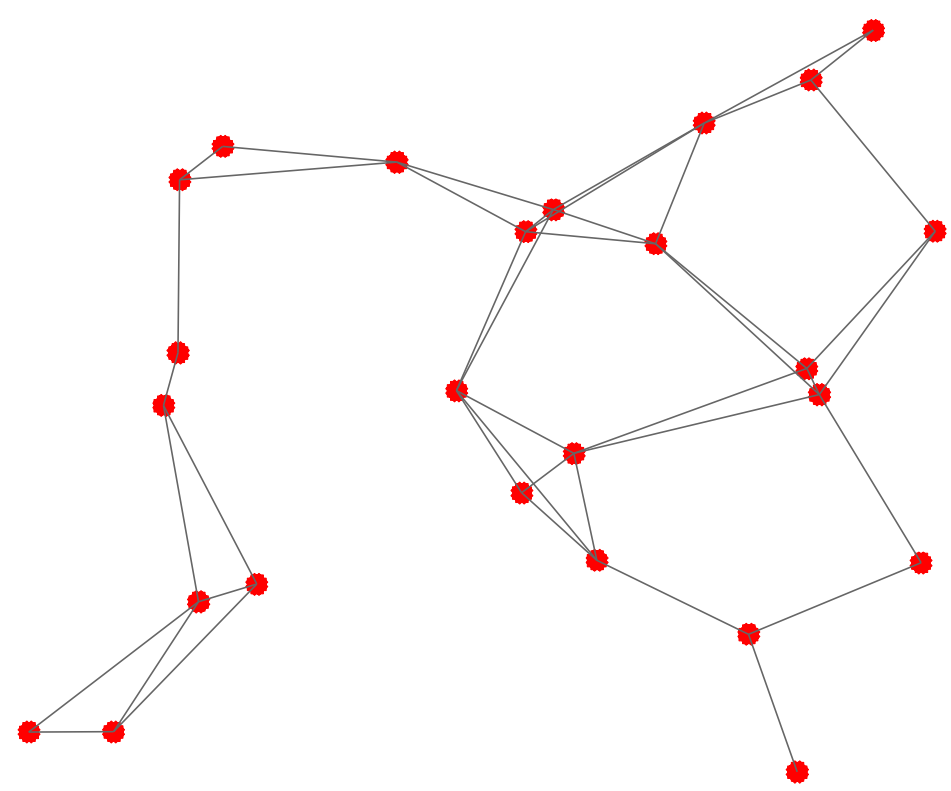

Figura 2. GD de uma rede aleatória com 25 nós.

Esta rede foi submetida ao algoritmo proposto, $K$-means-net, considerando $K=3$. A Figura 3 mostra a mesma rede após ser submetida ao K-means-net e a distribuição dos clusters. Os nós representados por são os nós apontados como melhores candidatos a gateways para a rede. Os nós de um mesmo cluster possuem cores iguais. A maior distância em saltos de um GW desta rede, após passar pelo processo de clustering é 2 .

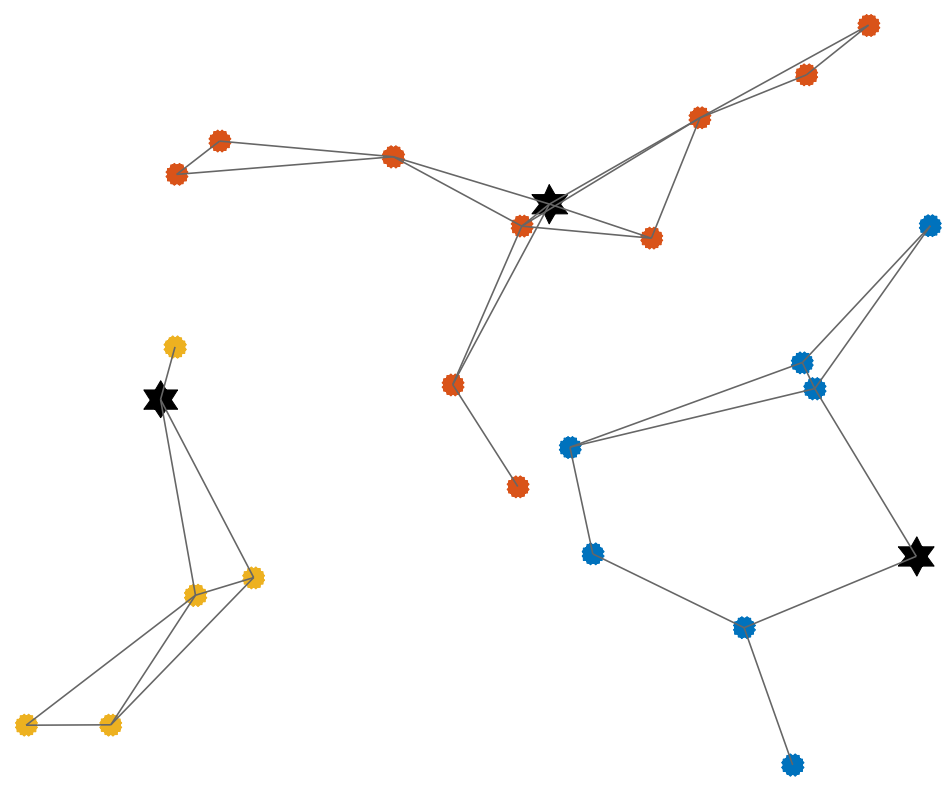

Figura 3. GD da rede aleatória de 25 nós com a divisão dos clusters e respectivos GWs.

Considerando agora uma rede aleatória e uniformemente distribuída com 50 nós, a Figura 4 representa seu respectivo GD, considerando $K=5$ e mesmo raio de cobertura que a rede da Figura 2. 


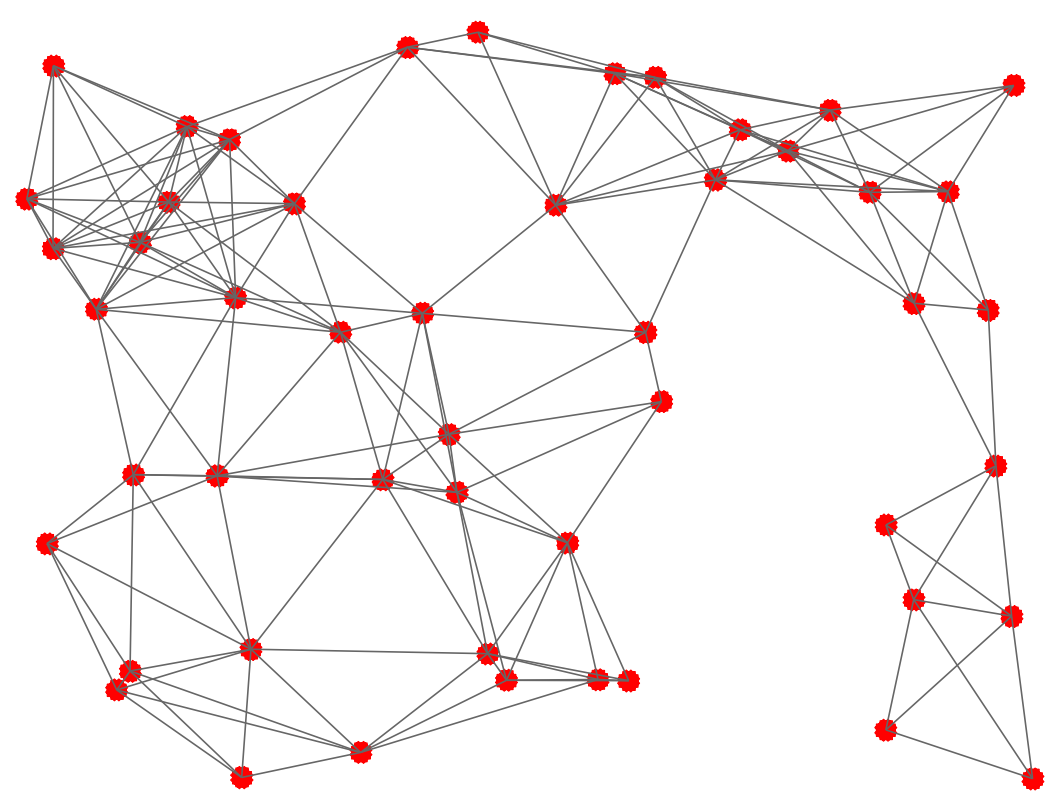

Figura 4. GD de uma rede aleatória com 50 nós.

Obtemos, após o processo de clustering, a rede ilustrada da Figura 5. A maior distância em saltos de um GW desta rede é, também, 2.

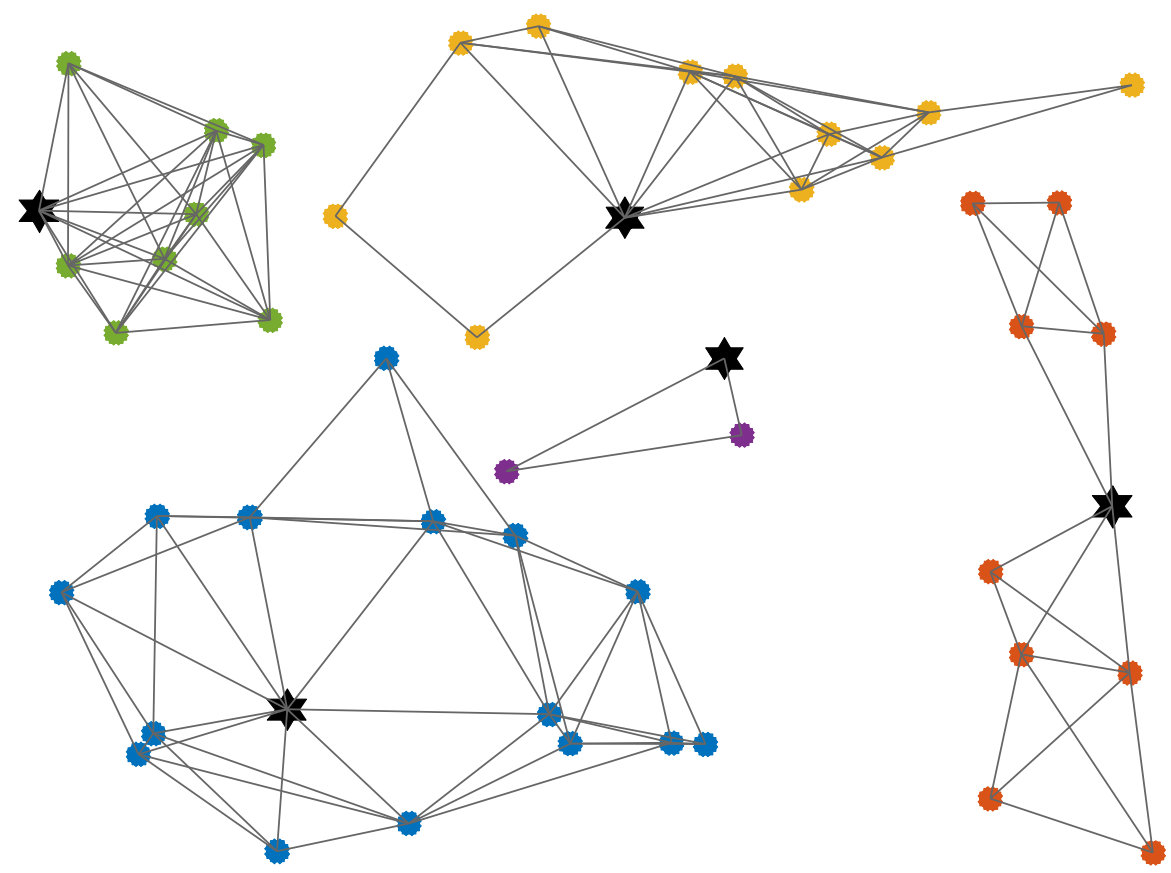

Figura 5. GD da rede aleatória de 50 nós com a divisão dos clusters e respectivos GWs.

Analisamos redes pequenas, com 25 e 50 nós, considerando 2, 3 e 5 gateways para a rede de 25 nós e 5,7 e 10 gateways para a rede de 50 nós. Também analisamos redes grandes, com 100 e 200 nós, considerando, agora, 10, 15 e 20 gateways para a rede composta por 100 nós e 20, 35 e 50 gateways para a rede com 200 nós. 
A Tabela 2 mostra a média dos parâmetros número de iterações, tempo de execução e máxima distância em saltos para 50 execuções do algoritmo K-means-net para as redes citadas anteriormente.

Tabela 2. Média das variações do tempo de execução, iterações e máximo salto com o aumento do número de gateways

\begin{tabular}{ccccc}
\hline Nós & Gateways & $\begin{array}{c}\text { Número médio } \\
\text { de iterações }\end{array}$ & $\begin{array}{c}\text { Tempo médio } \\
\text { de execução } \\
\text { (s) }\end{array}$ & $\begin{array}{c}\text { Máxima } \\
\text { distância média } \\
\text { (saltos) }\end{array}$ \\
\hline 25 & 2 & 5,72 & 0,096212 & 3,56 \\
25 & 3 & 5,90 & 0,105469 & 2,56 \\
25 & 5 & 6,36 & 0,111409 & 2,10 \\
50 & 5 & 8,78 & 0,132006 & 2,18 \\
50 & 7 & 9,04 & 0,141691 & 2,06 \\
50 & 10 & 9,22 & 0,144339 & 2,04 \\
100 & 10 & 14,98 & 0,201001 & 2,00 \\
100 & 15 & 8,08 & 0,173505 & 1,94 \\
100 & 20 & 7,24 & 0,168442 & 1,68 \\
200 & 20 & 10,24 & 0,280234 & 1,76 \\
200 & 35 & 3,18 & 0,186043 & 1,06 \\
200 & 50 & 9,04 & 0,168149 & 1,04 \\
\hline
\end{tabular}

\section{DISCUSSAOO}

Através da Tabela 2 é possível observar que a variação do valor de $K$ acarreta variações no máximo salto entre GW e o nó, no tempo de execução do algoritmo e na quantidade de iterações necessárias para se chegar a uma resposta satisfatória. $O$ aumento do valor de $K \mathrm{e}$, consequentemente, o aumento do número de gateways espalhados pela rede diminui o tempo de execução e o número de iterações para redes grandes. Para redes pequenas, estes dois parâmetros aumentam.

Para redes menores, a quantidade de nós não redundantes (possíveis GWs) é maior. Por isso, quanto menor o valor de $K$ desejado mais iterações são necessárias para se determinar os gateways da rede e, consequentemente, o tempo de execução aumenta. Para redes grandes ocorre o oposto. A quantidade de nós não redundantes é menor e, consequentemente, achar os melhores candidatos a GWs na rede demanda menos iterações e menor tempo de execução do algoritmo. 
O parâmetro que mantêm o mesmo padrão de variação é o máximo salto. Em todos os casos, o aumento do valor de $K$ minimiza ou torna aproximadamente constante o máximo salto da rede. Isso é esperado, uma vez que aumentar o número de $K$, aumenta o número de clusters e diminui a quantidade de nós em um mesmo cluster, o que acarreta a diminuição do maior salto. Essa diminuição do maior salto é exatamente o objetivo do nosso problema de otimização, que como demonstrado através da Tabela 2 e ilustrado pelas Figuras 3 e 5, foi satisfatoriamente alcançado.

\section{CONSIDERAÇOES FINAIS}

Este trabalho propõe a aplicação do algoritmo K-means-net, que trata da aplicação de um algoritmo inteligente ( $K$-means modificado) em conjunto com teoremas de substituição a uma rede de sensores sem fio de múltiplos saltos, a fim de encontrar os $K$ candidatos a gateways que minimizem a maior distância dos nós aos GWs.

O objetivo foi alcançado através das funções folha() e $t$ _step(), que encontram os GWs reduzindo o espaço de busca de cada cluster através do aumento de $t$ e, resultando apenas em nós não redundantes, ou seja, nós que são bons candidatos a serem GWs.

Os resultados mostram que o algoritmo K-means-net é eficiente para dividir clusters e encontrar os $K$ bons candidatos a gateways dentro da RSSF. Infelizmente não é possível garantir GWs ótimos, contudo o algoritmo se mostrou eficiente, retornando boas respostas.

\section{AGRADECIMENTOS}

Os autores deste artigo agradecem à FAPEMIG (APQ-01366-16) e ao CNPq pelo apoio financeiro.

\section{REFERÉNCIAS}

ABDELKHALEK, Ons; KRICHEN, Saoussen; GUITOUNI, Adel. A genetic algorithm based decision support system for the multi-objective node placement problem in next wireless generation network. Applied Soft Computing. vol.33. 2015. p. 278-291.

HUANG, Shu-Quiang; ZHANG, Zhen; LI, Yang; LIU, Zhu-Song; LI, Young-Hui. Deployment optimization of multi-hop wireless networks based on substitution graph. Information Sciences. vol. 400-401. 2017. p. 129-141.

KANUNGO, Tapas; MOUNT, David M.; NETANYAHU, Nathan S.; PIATKO, Christine D.; SILVERMAN, Ruth; WU, Angela Y. An Efficient k-Means Clustering Algorithm: Analysis and Implementation. IEEE Trans. Pattern Anal. Mach. Intell. vol. 24. n. 7. 2002. p. 881-892.

LIN, Chun-Cheng. Dynamic router node placement in wireless mesh networks: A PSO 
approach with constriction coefficient and its convergence analysis. Information Sciences. vol. 232. 2013. p. 297-308.

LIN, Chun-Cheng; CHEN, Teng-Huei; CHIN, Hui-Hsin. Adaptive router node placement with gateway positions and QoS constraints in dynamic wireless mesh networks. Journal of Network and Computer Applications. vol. 74. 2016. p. 149-164.

MAGÁN-CARRIÓN, Roberto; RODRÍGUES-GÓMEZ, Rafael A.; CAMACHO, José; GARCÍA-TEODORO, Pedro. Optimal relay placement in multi-hop wireless networks. Ad Hoc Networks. vol. 23. 2016. p.23-36.

REBAI, Maher; BERRE, Matthieu Le; SNOUSSI, Hichem; HNAIEN, Faicel; KHOUKHI, Lyes. Sensor deployment optimization methods to achieve both coverage and connectivity in wireless sensor networks. Computers \& Operations Research. vol. 59. 2015. p. 11-21.

YOON, Y; KIM, Y. H. An Efficient Genetic Algorithm for Maximum Coverage Deployment in Wireless Sensor Networks. IEEE Transactions on Cybernetics. vol. 43. n. 5. 2013. p. 1473-1483. 